\title{
The ideal relativistic spinning gas: polarization and spectra
}

\author{
F. Becattini \\ Università di Firenze and INFN Sezione di Firenze, Florence, Italy \\ F. Piccinini \\ INFN Sezione di Pavia, Pavia, Italy
}

\begin{abstract}
We study the physics of the ideal relativistic rotating gas at thermodynamical equilibrium and provide analytical expressions of the momentum spectra and polarization vector for the case of massive particles with spin $1 / 2$ and 1 . We show that the finite angular momentum $\mathbf{J}$ entails an anisotropy in momentum spectra, with particles emitted orthogonally to $\mathbf{J}$ having, on average, a larger momentum than along its direction. Unlike in the non-relativistic case, the proper polarization vector turns out not to be aligned with the total angular momentum with a non-trivial momentum dependence.
\end{abstract}

\section{INTRODUCTION}

The thermodynamics of rotating systems is a subject of interest mainly in astrophysics, where spinning objects are compact stars. Also in nuclear physics, there have been noteworthy applications to the problem of multifragmentation $[1,2]$. In most those applications, either the non-relativistic approximation is used or the spin of particles is neglected, so that a full description of the relativistic case including spin degrees of freedom is still missing. However, recently, the microcanonical and grand-canonical partition function of an ideal relativistic quantum gas of particles with spin have been calculated [3] enforcing angular momentum conservation. Taking advantage of these results, in this paper we work out analytical expressions of the spectra and polarization of particles in a relativistic rotating gas with large (in $\hbar$ units) angular momentum. From a phenomenological point of view, these calculations might be of interest for the physics of relativistic heavy ion collisions, where the formation of a system with a large intrinsic angular momentum has been envisaged [4]. We will confine ourselves to the case of Boltzmann statistics, leaving the quantum statistics case to future work.

The paper is organized as follows: in Sect. II we discuss the statistical mechanics of an ideal relativistic gas with fixed, large angular momentum and show the equivalence with a rigidly rotating system; in Sect. III we analyze more in detail the relation between angular momentum and angular velocity in the limit of low rotational speed; in Sect. IV we calculate the inclusive momentum spectra of particles; finally, in Sect. V we obtain the expressions of the polarization vectors of massive particles with spin $1 / 2$ and 1 .

\section{Notation}

In this paper we adopt the natural units, with $\hbar=c=K=1$. Space-time linear transformations (translations, rotations, boosts) and $\mathrm{SL}(2, \mathrm{C})$ transformations are written in serif font, e.g. R, L. Operators in Hilbert space will be

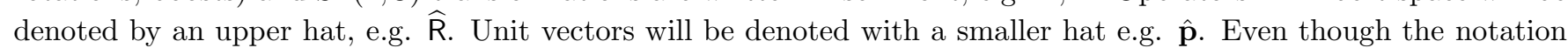
is unambiguous, we will make explicit mention of either possibility whenever confusion may arise.

\section{ROTATING RELATIVISTIC GAS AT EQUILIBRIUM}

In general, by rotating thermodynamical system we mean a system with fixed angular momentum in its rest frame. This is a good definition also in the relativistic case. It is well known that a classical system with non-vanishing intrinsic angular momentum can be at thermodynamical equilibrium only if the rotation is rigid [5], that is if a constant vector $\boldsymbol{\omega}$ exists such that the local collective velocity $\mathbf{v}$ is:

$$
\mathbf{v}=\boldsymbol{\omega} \times \mathbf{x}
$$

We will show that this conclusion holds for the relativistic gas, with the obvious inequality:

$$
\|\boldsymbol{\omega} \times \mathbf{x}\|<1
$$

In this section, we will develop a proof based on an ab initio statistical mechanics calculation while a proof based on an extension of Landau's argument for relativistic systems is given in Appendix B. 
In ref. [3], one of us derived the full expression of the microcanonical partition function, as well as its grand-canonical limit for large volumes, of a multi-species ideal relativistic gas with fixed angular momentum. In relativistic quantum mechanics, fixing the angular momentum means projecting onto irreducible states of the orthochronous Poincaré group $\mathrm{IO}(1,3)^{\uparrow}$ which, in fact, is a projection onto angular momentum states in the rest frame, where the total linear momentum vanishes. If, moreover, the intrinsic angular momentum is large (in $\hbar$ units), it can be treated as a classical vector $\mathbf{J}$ and the grand-canonical partition function reads [3]:

$$
\begin{aligned}
Z_{J} & =\operatorname{tr}\left\{\exp [(-\widehat{H}+\mu \widehat{Q}) / T] P_{\mathbf{J}} \mathrm{P}_{V}\right\} \\
& =\frac{2 J+1}{8 \pi^{2}} \int_{|\boldsymbol{\phi}|<\pi} \mathrm{d}^{3} \phi \mathrm{e}^{i \boldsymbol{\phi} \cdot \mathbf{J}} \exp \left[\sum_{j} \frac{\lambda_{j}}{(2 \pi)^{3}} \int_{V} \mathrm{~d}^{3} \mathrm{x} \int \mathrm{d}^{3} \mathrm{p} \mathrm{e}^{-\varepsilon_{j} / T} \mathrm{e}^{-i \boldsymbol{\phi} \cdot(\mathbf{x} \times \mathbf{p})} \operatorname{tr} D^{S_{j}}\left(\mathrm{R}_{\hat{\boldsymbol{\phi}}}(\phi)\right)\right]
\end{aligned}
$$

where $T$ is the temperature and $V$ the volume; $\varepsilon_{j}$ is the energy, $\lambda_{j}$ the fugacity and $S_{j}$ the spin of the $j$ th particle species; $D^{S_{j}}\left(\mathrm{R}_{\hat{\phi}}(\phi)\right)$ is the matrix of the irreducible representation $S_{j}$ of $\mathrm{SU}(2)$ transformation $\mathrm{R}$ with axis $\hat{\phi}$ and angle $\phi=\|\phi\|$. The operator $\widehat{H}$ is the hamiltonian, $\widehat{Q}$ is a generic conserved charge operator and $\mu$ the relevant chemical potential; the operator $\mathrm{P}_{\mathbf{J}}$ is the projector onto a fixed angular momentum while:

$$
\mathrm{P}_{V}=\sum_{h_{V}}\left|h_{V}\right\rangle\left\langle h_{V}\right|
$$

is the projector onto localized states $\left|h_{V}\right\rangle$, which form a complete set of quantum states for the system in the finite region $V$. This projector is needed in eq. (3) for the trace operation to be a properly defined one, i.e. involving a basis of the full Hilbert space [3].

The formula (3) applies to a system with large $V$ and $J$ and the integrand function turns out to be significant only in a region where $\phi=\|\phi\| \ll 1$. Since both $J$ and $V$ are large, it is then possible to make a saddle-point expansion of the integral (3). To do this, we first have to continue the integration variables to the complex plane and, in view of the spherical symmetry of the domain, the obvious choice is to take the spherical coordinates of the vector $\phi$, namely its magnitude $\phi$ along with its polar and azimuthal angles. Then, we have to solve the complex vector equation:

$$
\nabla_{\boldsymbol{\phi}}\left[i \boldsymbol{\phi} \cdot \mathbf{J}+\sum_{j} \frac{\lambda_{j}}{(2 \pi)^{3}} \int_{V} \mathrm{~d}^{3} \mathrm{x} \int \mathrm{d}^{3} \mathrm{p} \operatorname{tr} D^{S_{j}}\left(\mathrm{R}_{\hat{\boldsymbol{\phi}}}(\phi)\right) \mathrm{e}^{-\varepsilon_{j} / T} \mathrm{e}^{-i \phi \cdot(\mathbf{x} \times \mathbf{p})}\right]=0
$$

leading to:

$$
\begin{aligned}
\mathbf{J} & =\sum_{j} \frac{\lambda_{j}}{(2 \pi)^{3}} \int_{V} \mathrm{~d}^{3} \mathrm{x} \int \mathrm{d}^{3} \mathrm{p} \operatorname{tr} D^{S_{j}}\left(\mathrm{R}_{\hat{\boldsymbol{\phi}}}(\phi)\right)(\mathbf{x} \times \mathbf{p}) \mathrm{e}^{-\varepsilon_{j} / T} \mathrm{e}^{-i \phi \cdot(\mathbf{x} \times \mathbf{p})} \\
& +\sum_{j} \frac{\lambda_{j}}{(2 \pi)^{3}} \int_{V} \mathrm{~d}^{3} \mathrm{x} \int \mathrm{d}^{3} \mathrm{p}\left[i \nabla_{\boldsymbol{\phi}} \operatorname{tr} D^{S_{j}}\left(\mathrm{R}_{\hat{\boldsymbol{\phi}}}(\phi)\right)\right] \mathrm{e}^{-\varepsilon_{j} / T} \mathrm{e}^{-i \boldsymbol{\phi} \cdot(\mathbf{x} \times \mathbf{p})} \\
& \equiv \mathbf{L}(\boldsymbol{\phi})+\mathbf{S}(\boldsymbol{\phi})
\end{aligned}
$$

the reason for naming the two integral terms as $\mathbf{L}(\phi)$ and $\mathbf{S}(\phi)$ will become clear later on. We first observe that, if the equation (4) is solved by $\phi, \hat{\phi}$, then also $-\phi^{*}, \hat{\phi}^{*}$ is a solution. This can be easily checked by taking into account that the trace depends only on $\phi$ and can be written as a sum of exponentials $\exp [$ in $\phi]$, being $n$ an integer. Therefore, we will look for one solution enforcing $\phi=-\phi^{*}$ and $\hat{\phi}=\hat{\phi}^{*}$, that is with a real unit vector $\hat{\phi}$ and an imaginary magnitude $\phi$.

Assuming that one solution exists and defining the real vector $\boldsymbol{\omega} \equiv-i T \boldsymbol{\phi}$, we can write the grand-canonical partition function at the lowest order of the saddle-point expansion as:

$$
Z_{J} \propto \exp [-\boldsymbol{\omega} \cdot \mathbf{J} / T] \exp \left[\sum_{j} \frac{\lambda_{j}}{(2 \pi)^{3}} \int_{V} \mathrm{~d}^{3} \mathrm{x} \int \mathrm{d}^{3} \mathrm{p} \operatorname{tr} D^{S_{j}}\left(\mathrm{R}_{\hat{\boldsymbol{\omega}}}(i \omega / T)\right) \mathrm{e}^{-\varepsilon_{j} / T} \mathrm{e}^{\boldsymbol{\omega} \cdot(\mathbf{x} \times \mathbf{p}) / T}\right]
$$

The function:

$$
Z_{\omega}=\exp \left[\sum_{j} \frac{\lambda_{j}}{(2 \pi)^{3}} \int_{V} \mathrm{~d}^{3} \mathrm{x} \int \mathrm{d}^{3} \mathrm{p} \operatorname{tr} D^{S_{j}}\left(\mathrm{R}_{\hat{\boldsymbol{\omega}}}(i \omega / T)\right) \mathrm{e}^{-\varepsilon_{j} / T} \mathrm{e}^{\boldsymbol{\omega} \cdot(\mathbf{x} \times \mathbf{p}) / T}\right]
$$


is the partition function of an ideal relativistic gas (in the Boltzmann limit) rotating with an angular velocity $\boldsymbol{\omega}$. This can be shown explicitely for the spinless case by dividing the gas into small cells with volume $\Delta^{3} x$ and uniform velocity (1) and calculating the relevant grand-canonical partition function in the formalism of relativistic thermodynamics $[6]$ :

$$
Z_{\text {cell }}=\exp \left[\sum_{j} \frac{\lambda_{j}}{(2 \pi)^{3}} \Delta^{3} \mathrm{x} \int \mathrm{d}^{3} \mathrm{p} \mathrm{e}^{-\beta \cdot p}\right]
$$

where $\beta$ is the temperature four-vector:

$$
\beta=\frac{1}{T_{0} \sqrt{1-v^{2}}}(1, \mathbf{v})=\frac{1}{T_{0} \sqrt{1-v^{2}}}(1, \boldsymbol{\omega} \times \mathbf{x})
$$

$T_{0}$ being the local temperature, i.e. the temperature measured by a thermometer moving along with the cell. Note that with this equation, we are tacitly assuming that the partition function, which is a Lorentz-invariant quantity, in the accelerated rotating cell is the same as that in an inertial frame with its instantaneous origin and velocity ${ }^{1}$. This is consistent with the locality hypothesis [7] and the transformation law of energy in a rotating frame [7]:

$$
E^{\prime}=\gamma(E-\boldsymbol{\omega} \cdot \mathbf{L})=\gamma(E-\boldsymbol{\omega} \cdot(\mathbf{x} \times \mathbf{P}))
$$

being $\gamma=1 / \sqrt{1-\|\boldsymbol{\omega} \times \mathbf{x}\|^{2}}$. Therefore, with the identification:

$$
T=T_{0} \sqrt{1-v^{2}}=T_{0} \sqrt{1-\|\boldsymbol{\omega} \times \mathbf{x}\|^{2}}
$$

the partition function of the cell becomes:

$$
Z_{\text {cell }}=\exp \left[\sum_{j} \frac{\lambda_{j}}{(2 \pi)^{3}} \Delta^{3} \mathrm{x} \int \mathrm{d}^{3} \mathrm{p} \mathrm{e}^{-\varepsilon_{j} / T} \mathrm{e}^{\mathbf{p} \cdot(\boldsymbol{\omega} \times \mathbf{x}) / T}\right]
$$

The total partition function of the supposedly independent cells can now be obtained by multiplying expressions (12) for all cells and going to the limit of infinitesimal cells, implying:

$$
Z_{\omega}=\exp \left[\sum_{j} \frac{\lambda_{j}}{(2 \pi)^{3}} \int_{V} \mathrm{~d}^{3} \mathrm{x} \int \mathrm{d}^{3} \mathrm{p}^{-\varepsilon_{j} / T} \mathrm{e}^{\boldsymbol{\omega} \cdot(\mathbf{x} \times \mathbf{p}) / T}\right]
$$

which is the (7) for spinless particles.

Therefore, unlike in the non-relativistic case, the proper temperature $T_{0}$ in a spinning relativistic system at equilibrium depends on the distance from the rotation axis, an effect pointed out by Israel [8]. However, the thermal equilibrium is characterized by a uniform temperature $T$ as measured by the observer; another proof of this statement based on simple arguments can be found in Appendix B.

The partition function $Z_{\omega}$ defines an ensemble, that we can define as rotational grand-canonical where the angular velocity is fixed and the intrinsic angular momentum $\mathbf{J}$ can fluctuate. On the other hand, the grand-canonical partition function with fixed intrinsic angular momentum $\mathbf{J}$ defines an ensemble that can be called micro-rotational grand-canonical. The above definitions are inspired of the more familiar canonical-microcanonical duality, according to whether $T$ or $E$ are fixed.

Pursuing the analogy, it is expected that these two ensembles become equivalent in the thermodynamic limit as far as the calculation of first-order moments of statistical distributions is concerned. In fact, neglecting the constant small factors multiplying $Z_{J}$, it can be seen from (6) that $\log Z_{\omega}$ is essentially the Legendre transform of $\log Z_{J}$ with respect to the total angular momentum $\mathbf{J}$ :

$$
\log Z_{\omega}=\log Z_{J}+\frac{\boldsymbol{\omega} \cdot \mathbf{J}}{T}=\log Z_{J}+\mathbf{J} \cdot \frac{\partial}{\partial \mathbf{J}} \log Z_{J}
$$

\footnotetext{
${ }^{1}$ Note that thermodynamic equilibrium is possible in a rotating cell because its acceleration is stationary
} 
The saddle-point equation (5) for $\phi=i \boldsymbol{\omega} / T$ is simply the inverse Legendre transformation of (14):

$$
\begin{aligned}
\mathbf{J} & =\sum_{j} \frac{\lambda_{j}}{(2 \pi)^{3}} \int_{V} \mathrm{~d}^{3} \mathrm{x} \int \mathrm{d}^{3} \mathrm{p} \operatorname{tr} D^{S_{j}}\left(\mathrm{R}_{\hat{\boldsymbol{\omega}}}(i \omega / T)\right)(\mathbf{x} \times \mathbf{p}) \mathrm{e}^{-\varepsilon_{j} / T} \mathrm{e}^{\boldsymbol{\omega} \cdot(\mathbf{x} \times \mathbf{p}) / T} \\
& +\sum_{j} \frac{\lambda_{j}}{(2 \pi)^{3}} \int_{V} \mathrm{~d}^{3} \mathrm{x} \int \mathrm{d}^{3} \mathrm{p}\left[\frac{\partial}{\partial \omega / T} \operatorname{tr} D^{S_{j}}\left(\mathrm{R}_{\hat{\boldsymbol{\omega}}}(i \omega / T)\right)\right] \hat{\boldsymbol{\omega}} \mathrm{e}^{-\varepsilon_{j} / T} \mathrm{e}^{\boldsymbol{\omega} \cdot(\mathbf{x} \times \mathbf{p}) / T} \\
& =\mathbf{L}(\boldsymbol{\omega} / T)+\mathbf{S}(\boldsymbol{\omega} / T)=\frac{\partial}{\partial \boldsymbol{\omega} / T} \log Z_{\omega}
\end{aligned}
$$

Therefore, the saddle point equation (15) relates angular velocity $\boldsymbol{\omega}$ and angular momentum $\mathbf{J}$ and applies to relativistic as well as non-relativistic gases. It expresses the conservation of angular momentum, in the sense that the total angular momentum in the rotational grand-canonical ensemble on the right hand side, where the two contributions of orbital and spin can be clearly identified, equals the initial conserved total angular momentum $\mathbf{J}$ on the left hand side. It is worth pointing out that for macroscopic systems, the contribution of the orbital angular momentum is predominant. This can be seen from eq. (15) noting that the integral defining the total orbital angular momentum is scaled by a factor $\|\mathbf{x} \times \mathbf{p}\|$ with respect to the spin angular momentum. Since this is generally much larger than 1, for macroscopic distances and not too low temperatures, we usually have $\|\mathbf{L}\| \gg\|\mathbf{S}\|$ and the spin angular momentum can be neglected. Yet, for not too large systems, the spin angular momentum contribution can be sizeable. There is also a remarkable difference between them concerning their dependence on the size of the sytem: the spin angular momentum is properly extensive, i.e. it increase linearly with the volume, while the orbital angular momentum is not because of the factor $\|\mathbf{x} \times \mathbf{p}\|$. The lack of extensivity of rotating systems makes the thermodynamic limit essentially irrelevant and dictates a finite-size treatment. From a completely equivalent point of view of the comoving frame, we can state that the rotating system is non-extensive because of the presence of a long-range centrifugal potential.

Finally, it can be shown that in the rotational grand-canonical ensemble each microstate with total energy $E$, total charge $Q$ and total angular momentum $\mathbf{J}$ has a probability (in the frame where $\langle\mathbf{P}\rangle=0$ ):

$$
p(T, \mu, \boldsymbol{\omega}) \propto \exp [-E / T+\mu Q / T+\boldsymbol{\omega} \cdot \mathbf{J} / T]
$$

and, consequently, rotational grand-canonical partition function can be written as:

$$
Z_{\omega}=\operatorname{tr}\left\{\exp [(-\widehat{H}+\mu \widehat{Q}+\boldsymbol{\omega} \cdot \widehat{\mathbf{J}}) / T] \mathrm{P}_{V}\right\}
$$

where $\widehat{\mathbf{J}}$ is the total angular momentum operator. This statement holds provided that $\omega / T \ll 1$ so that terms of the order $(\omega / T)^{2}$ and higher can be neglected; only in this case is the formula (17) equivalent to (7). Indeed, working out the trace (17) for an ideal gas involves the calculation of matrix elements such as:

$$
\sum_{\sigma}\left\langle p, \sigma\left|\exp [\boldsymbol{\omega} \cdot \widehat{\mathbf{J}} / T] \mathrm{P}_{V}\right| p, \sigma\right\rangle=\sum_{\sigma}\left\langle p, \sigma\left|\hat{\mathrm{R}}_{\hat{\boldsymbol{\omega}}}(i \omega / T) \mathrm{P}_{V}\right| p, \sigma\right\rangle
$$

where $|p, \sigma\rangle$ are single particle states with four-momentum $p$ and spin projection $\sigma$ (see Sect. 5 for extended discussion) and the rotation of an imaginary angle $i \omega / T$ has been introduced. The above single-particle trace has been calculated in ref. [3]:

$$
\sum_{\sigma}\left\langle p, \sigma\left|\hat{\mathrm{R}}_{\hat{\boldsymbol{\omega}}}(i \omega / T] \mathrm{P}_{V}\right| p, \sigma\right\rangle=\frac{1}{(2 \pi)^{3}} \int_{V} \mathrm{~d}^{3} \mathrm{x} \mathrm{e}^{i \mathbf{x} \cdot\left(\mathbf{p}-\mathrm{R}_{\hat{\boldsymbol{\omega}}}(i \omega / T)^{-1} \mathbf{p}\right)} \operatorname{tr} D^{S}\left(\mathrm{R}_{\hat{\boldsymbol{\omega}}}(i \omega / T)\right)\left\langle 0\left|\mathrm{P}_{V}\right| 0\right\rangle
$$

The vacuum expectation value of $\mathrm{P}_{V}$ is immaterial and also becomes 1 in the large volume limit. Now, since:

$$
\mathrm{R}_{\hat{\boldsymbol{\omega}}}(\psi) \mathbf{v}=\mathbf{v} \cos \psi+(\hat{\mathbf{n}} \times \mathbf{v}) \sin \psi+(1-\cos \psi) \mathbf{v} \cdot \hat{\mathbf{n}} \hat{\mathbf{n}}
$$

we have:

$$
\left.\mathbf{x} \cdot\left(\mathbf{p}-\mathrm{R}_{\hat{\boldsymbol{\omega}}}(i \omega / T)^{-1} \mathbf{p}\right)\right] \simeq i \frac{\omega}{T} \mathbf{x} \cdot(\boldsymbol{\omega} \times \mathbf{p})
$$

only if $\omega / T$ is sufficiently smaller than 1 , so that cosine terms in (20) can be approximated with 1 and sine terms with their argument. In this case, eq. (19) turns into, for large $V$ :

$$
\sum_{\sigma}\left\langle p, \sigma\left|\hat{\mathrm{R}}_{\hat{\boldsymbol{\omega}}}(i \omega / T) \mathrm{P}_{V}\right| p, \sigma\right\rangle \simeq \frac{1}{(2 \pi)^{3}} \int_{V} \mathrm{~d}^{3} \mathrm{x} \mathrm{e}^{\boldsymbol{\omega} \cdot(\mathbf{x} \times \mathbf{p}) / T} \operatorname{tr} D^{S}\left(\mathrm{R}_{\hat{\boldsymbol{\omega}}}(i \omega / T)\right)
$$

It is then straightforward to show that the final expression of (17) is just (7). 


\section{MOMENTS OF INERTIA}

In this section, we will study more in detail the saddle-point equation (5) or its equivalent form (15). This is a vector equation in $\boldsymbol{\omega}$, whose solution depends, besides $T$ and $\mathbf{J}$, on the shape and size of the system.

If the total angular momentum $\mathbf{J}$ is a simmetry axis of $V$, then $\mathbf{J}$ and $\boldsymbol{\omega}$ must be parallel (see Fig. 1). This can be proved by applying an arbitrary rotation of an angle $\psi$ around $\mathbf{J}$, let $\mathbf{R}_{\hat{\mathbf{J}}}(\psi)$, to both sides of eq. (15). Since $R(\mathbf{x} \times \mathbf{p})=R \mathbf{x} \times R \mathbf{p}$, defining new integration variables $\mathbf{x}^{\prime}=R \mathbf{x}$ and $\mathbf{p}^{\prime}=R \mathbf{p}$, taking into account that $\boldsymbol{\omega} \cdot \mathrm{R}^{-1}\left(\mathbf{x}^{\prime} \times \mathbf{p}^{\prime}\right)=\mathrm{R} \boldsymbol{\omega} \cdot\left(\mathbf{x}^{\prime} \times \mathbf{p}^{\prime}\right)$ and being both momentum and spacial domains invariant by rotation around $\mathbf{J}$, we can conclude that, if $\boldsymbol{\omega}$ is a solution of the eq. (4), $\mathbf{R}_{\hat{\mathbf{J}}}(\psi) \boldsymbol{\omega}$ is also a solution for any $\psi$. Therefore, $\mathbf{R}_{\hat{\mathbf{J}}}(\psi) \boldsymbol{\omega}$ should coincide with $\boldsymbol{\omega}$ for the equation to be well behaved. If this is the case, $\boldsymbol{\omega}$ is parallel to $\mathbf{J}$ and the orbital term $\mathbf{L}(\boldsymbol{\omega} / T)$ should be also parallel to $\boldsymbol{\omega}$. In this case, the eq. (15) essentially reduces to a scalar one:

$$
\begin{aligned}
& J=\sum_{j} \frac{\lambda_{j}}{(2 \pi)^{3}} \int_{V} \mathrm{~d}^{3} \mathrm{x} \int \mathrm{d}^{3} \mathrm{p} \operatorname{tr} D^{S_{j}}\left(\mathrm{R}_{\hat{\boldsymbol{\omega}}}(i \omega / T)\right) \hat{\mathbf{J}} \cdot(\mathbf{x} \times \mathbf{p}) \mathrm{e}^{-\varepsilon_{j} / T} \mathrm{e}^{\omega \hat{\mathbf{J}} \cdot(\mathbf{x} \times \mathbf{p}) / T} \\
& +\sum_{j} \frac{\lambda_{j}}{(2 \pi)^{3}} \int_{V} \mathrm{~d}^{3} \mathrm{x} \int \mathrm{d}^{3} \mathrm{p}\left[\frac{\partial}{\partial \omega / T} \operatorname{tr} D^{S_{j}}\left(\mathrm{R}_{\hat{\boldsymbol{\omega}}}(i \omega / T)\right)\right] \mathrm{e}^{-\varepsilon_{j} / T} \mathrm{e}^{\omega \hat{\mathbf{J}} \cdot(\mathbf{x} \times \mathbf{p}) / T}
\end{aligned}
$$

which can be solved numerically.

Let us now introduce a length $R$ related to the size of the system; it can be, e.g. the maximal distance of a point of the set $V$ from the rotation axis. An analytic solution of the general equation (15) can be obtained for small values of $\omega R \mathrm{p} / T$. In fact, under this circumstance, the exponential can be approximated as:

$$
\exp [\boldsymbol{\omega} \cdot(\mathbf{x} \times \mathbf{p}) / T] \simeq 1+\boldsymbol{\omega} \cdot(\mathbf{x} \times \mathbf{p}) / T
$$

This condition leads to different requirements for a non-relativistic and an ultrarelativistic gas (being $p=\mathcal{O}(\sqrt{m T}$ ) with $m \gg T$ and $p=\mathcal{O}(T)$ respectively), yet both imply that $\omega R \ll 1$. If approximation (23) applies, the two terms $\mathbf{L}$ and $\mathbf{S}$ reduce to, at the first order in $\omega$ :

$$
\begin{aligned}
& \mathbf{L} \simeq \sum_{j} \frac{\lambda_{j}}{(2 \pi)^{3}} \int_{V} \mathrm{~d}^{3} \mathrm{x} \int \mathrm{d}^{3} \mathrm{p}\left(2 S_{j}+1\right)(\mathbf{x} \times \mathbf{p}) \frac{\boldsymbol{\omega}}{T} \cdot(\mathbf{x} \times \mathbf{p}) \mathrm{e}^{-\varepsilon_{j} / T} \\
& \mathbf{S} \simeq \sum_{j} \frac{\lambda_{j}}{(2 \pi)^{3}} \int_{V} \mathrm{~d}^{3} \mathrm{x} \int \mathrm{d}^{3} \mathrm{p} \frac{S_{j}\left(S_{j}+1\right)\left(2 S_{j}+1\right)}{3} \mathrm{e}^{-\varepsilon_{j} / T} \frac{\boldsymbol{\omega}}{T}
\end{aligned}
$$

By expanding the scalar and vector products in the integral of the orbital angular momentum and carrying out simple algebraic calculations, one obtains:

$$
L_{i}=\sum_{k=1}^{3} \sum_{j}\left\langle n_{j}\right\rangle \frac{\left\langle p_{j}^{2}\right\rangle}{3 T} \int_{V} \mathrm{~d}^{3} \mathrm{x}\left(r^{2} \delta_{i k}-\mathrm{x}_{i} \mathrm{x}_{k}\right) \omega_{k}
$$

where $r^{2}=\sum_{i=1}^{3} \mathrm{x}_{i}^{2}$ and:

$$
\left\langle n_{j}\right\rangle=\frac{\lambda_{j}\left(2 S_{j}+1\right)}{(2 \pi)^{3}} \int \mathrm{d}^{3} \mathrm{p}^{-\varepsilon_{j} / T}
$$

is the mean density of the species $j$ in the Boltzmann approximation and without angular momentum constraint, while:

$$
\left\langle p_{j}^{2}\right\rangle=\frac{1}{\left\langle n_{j}\right\rangle} \frac{\lambda_{j}\left(2 S_{j}+1\right)}{(2 \pi)^{3}} \int \mathrm{d}^{3} \mathrm{p} \mathrm{p}^{2} \mathrm{e}^{-\varepsilon_{j} / T}
$$

is the mean squared momentum of the particle $j$ under the same approximations.

The relation between $\mathbf{L}$ and $\boldsymbol{\omega}(25)$ resembles the classical linear relation between angular velocity and angular momentum defining the inertia tensor I. Indeed, in the non-relativistic limit, this is precisely what one gets from (25) because $\left\langle p_{j}^{2}\right\rangle=3 m_{j} T$ and, consequently:

$$
L_{i}=\sum_{k=1}^{3} \sum_{j} m_{j}\left\langle n_{j}\right\rangle \int_{V} \mathrm{~d}^{3} \mathrm{x}\left(r^{2} \delta_{i k}-\mathrm{x}_{\mathrm{i}} \mathrm{x}_{\mathrm{k}}\right) \omega_{k}=\sum_{k=1}^{3} I_{i k} \omega_{k}
$$


Hence the expression in (25):

$$
I_{i k}=\sum_{j}\left\langle n_{j}\right\rangle \frac{\left\langle p_{j}^{2}\right\rangle}{3 T} \int_{V} \mathrm{~d}^{3} \mathrm{x}\left(r^{2} \delta_{i k}-\mathrm{x}_{i} \mathrm{x}_{k}\right)
$$

turns out to be the right generalization of the inertia tensor for a relativistic gas. Like in the classical case, if the system (which is homogeneous for small $\omega$ ) is symmetric around its rotation axis, then $\mathbf{L}$ is parallel to $\boldsymbol{\omega}$ and the proportionality constant is just the moment of inertia with respect to that axis.

Unlike in the classical case, for non-macroscopic systems there is also a potentially non-negligible spin contribution. In this case, the full relation between $\mathbf{J}$ and $\boldsymbol{\omega}$ reads:

$$
J_{i}=\sum_{k=1}^{3} \sum_{j}\left\langle n_{j}\right\rangle\left[\frac{\left\langle p_{j}^{2}\right\rangle}{3 T} \int_{V} \mathrm{~d}^{3} \mathrm{x}\left(r^{2} \delta_{i k}-\mathrm{x}_{i} \mathrm{x}_{k}\right)+V \frac{S_{j}\left(S_{j}+1\right)}{3 T} \delta_{i k}\right] \omega_{k}
$$

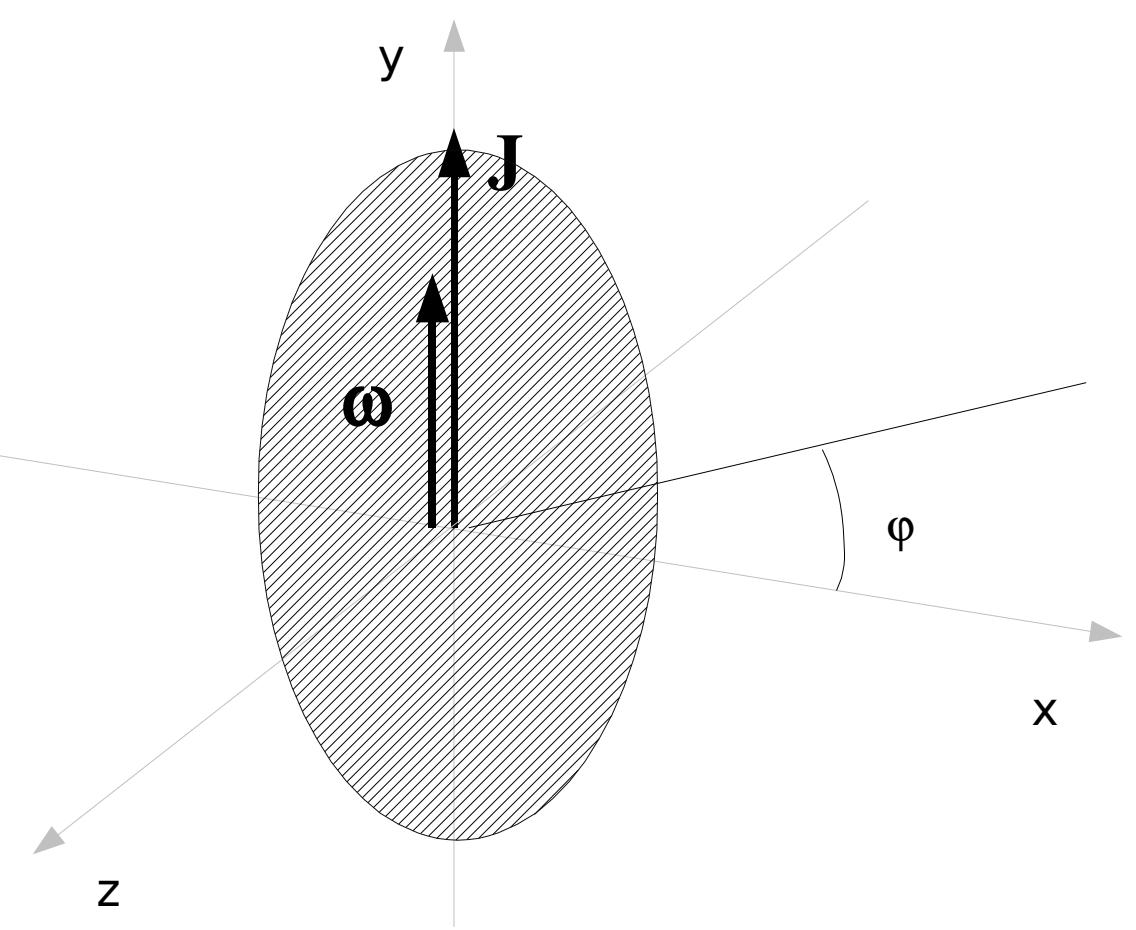

FIG. 1: For an axially symmetric system around the angular momentum direction, $\boldsymbol{\omega}$ is parallel to $\mathbf{J}$. Also shown the coordinates chosen to describe spectra in Sect. IV.

\section{SPECTRA AND ANISOTROPIES}

The impact of a finite angular momentum on particle spectra in a relativistic gas was considered by Hagedorn [9] who pointed out that a large value of the intrinsic angular momentum involves a peculiar anisotropy. Here, we will provide the most general expressions of the momentum spectrum of particles in an equilibrated rotating system with fixed (classical) angular momentum $\mathbf{J}$. This can be obtained directly, for the $j$ th particle species, from the partition function (3):

$$
\left\langle\frac{\mathrm{d} n_{j}}{\mathrm{~d}^{3} \mathrm{p}}\right\rangle=\frac{1}{Z_{J}} \operatorname{tr}\left\{\frac{\mathrm{d} \widehat{n}_{j}}{\mathrm{~d}^{3} \mathrm{p}} \exp [(-\widehat{H}+\mu \widehat{Q}) / T] P_{J} \mathrm{P}_{V}\right\}
$$

where $\mathrm{d} \widehat{n}_{j} / \mathrm{d}^{3} \mathrm{p}$ is the momentum spectrum operator. The above expression can be rewritten as the functional derivative with respect to $\alpha(\mathbf{p})$ of:

$$
Z_{J}[\alpha(\mathbf{p})]=\operatorname{tr}\left\{\exp \left[(-\widehat{H}+\mu \widehat{Q}) / T+\int \mathrm{d}^{3} \mathrm{p} \alpha(\mathbf{p}) \frac{\mathrm{d} \widehat{n}_{j}}{\mathrm{~d}^{3} \mathrm{p}}\right] P_{\mathbf{J}} \mathrm{P}_{V}\right\}
$$


in $\alpha(\mathbf{p})=0$. Since, for an ideal gas:

$$
\widehat{H}=\sum_{j} \int \mathrm{d}^{3} \mathrm{p} \varepsilon_{j}(\mathbf{p}) \frac{\mathrm{d} \widehat{n}_{j}}{\mathrm{~d}^{3} \mathrm{p}}
$$

the functional $Z_{J}[\alpha(\mathbf{p})]$ can be simply obtained from eq. (3) replacing $\varepsilon_{j} / T$ with $\varepsilon_{j} / T+\alpha(\mathbf{p})$. The functional derivative then turns out to be:

$$
\begin{aligned}
\left\langle\frac{\mathrm{d} n_{j}}{\mathrm{~d}^{3} \mathrm{p}}\right\rangle & =\left.\frac{\delta}{\delta \alpha(\mathbf{p})} \log Z_{J}[\alpha(\mathbf{p})]\right|_{\alpha(\mathbf{p})=0}=\frac{1}{Z_{J}} \frac{2 J+1}{8 \pi^{2}} \frac{\lambda_{j}}{(2 \pi)^{3}} \int_{V} \mathrm{~d}^{3} \mathrm{xe}^{-\varepsilon_{j} / T} \int_{|\boldsymbol{\phi}|<\pi} \mathrm{d}^{3} \phi \mathrm{e}^{i \boldsymbol{\phi} \cdot(\mathbf{J}-\mathrm{x} \times \mathrm{p})} \operatorname{tr} D^{S_{j}}\left(\mathrm{R}_{\hat{\boldsymbol{\phi}}}(\phi)\right) \\
& \times \exp \left[\sum_{k} \frac{\lambda_{k}}{(2 \pi)^{3}} \int_{V} \mathrm{~d}^{3} \mathrm{x} \int \mathrm{d}^{3} \mathrm{p}^{-\varepsilon_{k} / T} \mathrm{e}^{-i \boldsymbol{\phi} \cdot(\mathbf{x} \times \mathbf{p})} \operatorname{tr} D^{S_{k}}\left(\mathrm{R}_{\hat{\boldsymbol{\phi}}}(\phi)\right)\right]
\end{aligned}
$$

Similarly to eq. (3), it is possible to make a saddle-point expansion of the above integral. If the orbital angular momentum of the particle is much lower than the total angular momentum, i.e. $\|\mathbf{x} \times \mathbf{p}\| \ll J$, the former can be neglected in the saddle-point equation which is then essentially the same as that for the partition function, eq. (4). This condition amounts to take $\exp [-i \phi \cdot(\mathbf{x} \times \mathbf{p})]$ as a constant factor and it is usually met except for momenta larger than $J / R, R$ being the size of the system. In this case, the formula (32) becomes, at the leading order:

$$
\left\langle\frac{\mathrm{d} n_{j}}{\mathrm{~d}^{3} \mathrm{p}}\right\rangle=\frac{\lambda_{j}}{(2 \pi)^{3}} \operatorname{tr} D^{S_{j}}\left(\mathrm{R}_{\hat{\boldsymbol{\omega}}}(i \omega / T)\right) \int_{V} \mathrm{~d}^{3} \mathrm{x}^{-\varepsilon_{j} / T} \mathrm{e}^{\boldsymbol{\omega} \cdot(\mathbf{x} \times \mathbf{p}) / T}
$$

which is, of course, the spectrum in the rotational grand-canonical ensemble in the Boltzmann limit.

It is of some interest to derive the shape of the spectra in cylindrical momentum coordinates $p_{T}=\sqrt{p_{x}^{2}+p_{y}^{2}}$, azimuthal angle $\varphi$ and rapidity $y=1 / 2 \log \left[\left(p_{z}+\varepsilon\right) /\left(\varepsilon-p_{z}\right)\right]$ when the angular momentum of the system is directed along the $y$ axis, i.e. $\mathbf{J}=J \hat{\mathbf{j}}$ and the system is rotationally symmetric around $\mathbf{J}$. Because of this symmetry one has $\boldsymbol{\omega}=\omega \hat{\mathbf{j}}$ and the spectrum (33) reads:

$$
\left\langle\frac{\mathrm{d} n_{j}}{\mathrm{~d}^{3} \mathrm{p}}\right\rangle=\frac{\lambda_{j}}{(2 \pi)^{3}} \operatorname{tr} D^{S_{j}}\left(\mathrm{R}_{\hat{\boldsymbol{\omega}}}(i \omega / T)\right) \int_{V} \mathrm{~d}^{3} \mathrm{x}^{-\varepsilon_{j} / T} \mathrm{e}^{\boldsymbol{\omega} \cdot(\mathrm{x} \times \mathrm{p}) / T}
$$

In the new coordinates $p_{T}, \varphi, y$ the spectrum reads:

$$
\left\langle\frac{\mathrm{d} n_{j}}{p_{T} \mathrm{~d} p_{T} \mathrm{~d} \varphi \mathrm{d} y}\right\rangle=\frac{\lambda_{j} \operatorname{tr} D^{S_{j}}\left(\mathrm{R}_{\hat{\boldsymbol{\omega}}}(i \omega / T)\right)}{(2 \pi)^{3}} \int_{V} \mathrm{~d}^{3} \mathrm{x} m_{T} \cosh y \exp \left[-m_{T} \cosh y / T+\mathbf{p}_{T} \cdot(\boldsymbol{\omega} \times \mathbf{x})_{\perp} / T+m_{T}(\boldsymbol{\omega} \times \mathbf{x})_{\|} \sinh y / T\right]
$$

where $m_{T}=\sqrt{p_{T}^{2}+m_{j}^{2}}$, labels the longitudinal projection along the $z$ axis and $\perp$ the transverse projection onto the $x y$ plane.

With successive integrations of eq. (35) one obtains:

$$
\left\langle\frac{\mathrm{d} n_{j}}{p_{T} \mathrm{~d} p_{T} \mathrm{~d} \varphi}\right\rangle=\frac{\lambda_{j} \operatorname{tr} D^{S_{j}}\left(\mathrm{R}_{\hat{\boldsymbol{\omega}}}(i \omega / T)\right)}{4 \pi^{3}} \int_{V} \mathrm{~d}^{3} \mathrm{x} \frac{1}{\sqrt{1-(\boldsymbol{\omega} \times \mathbf{x})_{\|}^{2}}} m_{T} \mathrm{~K}_{1}\left(m_{T} \sqrt{1-(\boldsymbol{\omega} \times \mathbf{x})_{\|}^{2}} / T\right) \exp \left[\mathbf{p}_{T} \cdot(\boldsymbol{\omega} \times \mathbf{x})_{\perp} / T\right]
$$

and:

$$
\left\langle\frac{\mathrm{d} n_{j}}{p_{T} \mathrm{~d} p_{T}}\right\rangle=\frac{\lambda_{j} \operatorname{tr} D^{S_{j}}\left(\mathrm{R}_{\hat{\boldsymbol{\omega}}}(i \omega / T)\right)}{2 \pi^{2}} \int_{V} \mathrm{~d}^{3} \mathrm{x} \frac{1}{\sqrt{1-(\boldsymbol{\omega} \times \mathbf{x})_{\|}^{2}}} m_{T} \mathrm{~K}_{1}\left(m_{T} \sqrt{1-(\boldsymbol{\omega} \times \mathbf{x})_{\|}^{2}} / T\right) \mathrm{I}_{0}\left(p_{T}\left\|(\boldsymbol{\omega} \times \mathbf{x})_{\perp}\right\| / T\right)
$$

where $\mathrm{K}_{1}$ and $\mathrm{I}_{0}$ are McDonald and modified Bessel functions respectively. It is also possible to calculate the mean multiplicity by integrating (37), which yields:

$$
\left\langle n_{j}\right\rangle=\frac{\lambda_{j}}{2 \pi^{2}} \operatorname{tr} D^{S_{j}}\left(\mathrm{R}_{\hat{\boldsymbol{\omega}}}(i \omega / T)\right) \int_{V} \mathrm{~d}^{3} \mathrm{x} \frac{m^{2} T}{1-\|\boldsymbol{\omega} \times \mathbf{x}\|^{2}} \mathrm{~K}_{2}\left(m \sqrt{1-\|\boldsymbol{\omega} \times \mathbf{x}\|^{2}} / T\right)
$$

The formulae (35)-(38) yield as limiting cases for $\boldsymbol{\omega}=0$ the well known ones for the relativistic ideal Boltzmann gas. 
It is worth pointing out that the spectrum (35) features a non-trivial dependence on the momentum azimuthal angle $\varphi$ strongly sensitive to the ratio $\omega / T$. Introducing cylindrical coordinates $r, \Phi, z$ for the vector $\mathbf{x}$ and taking into account that $\boldsymbol{\omega}=\omega \hat{\mathbf{j}}$, eq. (36) can be written as:

$$
\left\langle\frac{\mathrm{d} n_{j}}{p_{T} \mathrm{~d} p_{T} \mathrm{~d} \varphi}\right\rangle=\frac{\lambda_{j} \operatorname{tr} D^{S_{j}}\left(\mathrm{R}_{\hat{\boldsymbol{\omega}}}(i \omega / T)\right)}{4 \pi^{3}} \int_{V} \mathrm{~d}^{3} \mathrm{x} \frac{1}{\sqrt{1-(\omega r \cos \Phi)^{2}}} m_{T} \mathrm{~K}_{1}\left(m_{T} \sqrt{1-(\omega r \cos \Phi)^{2}} / T\right) \exp \left[p_{T} \omega z \cos \varphi / T\right]
$$

which shows that particles emitted along the momentum axis (with $\varphi=\pi / 2$ ) have, on average, a lower momentum than those emitted orthogonally to it. Classically, this can be understood as an effect of centrifugal force.

The (39) is a periodic function in the momentum azimuthal angle $\varphi$, thus it can be Fourier expanded. Borrowing a notation commonly used in relativistic heavy ion physics, we will denote by $2 v_{k}$ the coefficients of this expansion, i.e. setting:

$$
\left\langle\frac{\mathrm{d} n_{j}}{p_{T} \mathrm{~d} p_{T} \mathrm{~d} \varphi}\right\rangle=\frac{1}{2 \pi}\left\langle\frac{\mathrm{d} n_{j}}{p_{T} \mathrm{~d} p_{T}}\right\rangle \sum_{k=0}^{\infty} 2 v_{2 k} \cos 2 k \varphi
$$

with $v_{0} \equiv 1$ we obtain:

$$
v_{2 k}=\frac{\int_{0}^{2 \pi}\left\langle\frac{\mathrm{d} n_{j}}{p_{T} \mathrm{~d} p_{T} \mathrm{~d} \varphi}\right\rangle \cos 2 k \varphi}{\int_{0}^{2 \pi}\left\langle\frac{\mathrm{d} n_{j}}{p_{T} \mathrm{~d} p_{T} \mathrm{~d} \varphi}\right\rangle}
$$

and, by using (39)

$$
v_{2 k}=\frac{\int_{V} \mathrm{~d}^{3} \mathrm{x} \frac{1}{\sqrt{1-(\omega r \cos \Phi)^{2}}} m_{T} \mathrm{~K}_{1}\left(m_{T} \sqrt{1-(\omega r \cos \Phi)^{2}} / T\right) I_{2 k}\left(p_{T} z \omega / T\right)}{\int_{V} \mathrm{~d}^{3} \mathrm{x} \frac{1}{\sqrt{1-(\omega r \cos \Phi)^{2}}} m_{T} \mathrm{~K}_{1}\left(m_{T} \sqrt{1-(\omega r \cos \Phi)^{2}} / T\right) I_{0}\left(p_{T} z \omega / T\right)}
$$

which shows that the Fourier coefficients are bound between 0 and 1 . For low $p_{T}$, the ratio between modified Bessel functions is such that $\mathrm{I}_{2 k} / \mathrm{I}_{0} \propto p_{T}^{2 k}$, while at large $p_{T}$ values, the ratio of modified Bessel functions tends to 1 and, as a consequence, all $v_{2 k} \rightarrow 1$.

\section{POLARIZATION}

If a gas has a net electric charge, an imbalance in the mean multiplicities of positive and negative particles is implied. Similarly, if a gas at thermodynamical equilibrium has a non-vanishing angular momentum (hence it is rigidly rotating, as we have seen in Sect. II) particles should have a net polarization along the direction of angular momentum. From the viewpoint of the comoving observer in the rotating frame, the hamiltonian in the rotating frame has a spin-rotation coupling term $[7,10]^{2}$. For the non-relativistic case, the calculation is straightforward as the polarization vector is the same in the observer and in the comoving frame, hence it can be carried out in the latter where the single-particle hamiltonian reads [10]:

$$
\widehat{h}_{\mathrm{rot}}=\widehat{h}_{\mathrm{obs}}-\boldsymbol{\omega} \cdot \widehat{\mathbf{j}}
$$

being $\widehat{\mathbf{j}}$ the total (orbital + spin) angular momentum operator of the particle. In fact, the problem we want to solve here is to calculate the polarization vector for a relativistic gas.

This problem is more difficult than for charge, energy or momentum because angular momentum is not a generator of an abelian group and this adds some complication. Particularly, from the point of view of statistical mechanics, one has to deal, as we will shortly see, with a spin density matrix with non-vanishing off-diagonal elements, unlike for momenta. From eq. (17), the density operator in the rotational grand-canonical ensemble reads:

$$
\widehat{\rho}_{\omega}=\frac{1}{Z_{\omega}} \exp [(-\widehat{H}+\mu \widehat{Q}+\boldsymbol{\omega} \cdot \widehat{\mathbf{J}}) / T] \mathrm{P}_{V}
$$

\footnotetext{
2 This phenomenon is well known for radio waves [7]
} 
while in the micro-rotational grand-canonical ensemble, from eq. (3):

$$
\widehat{\rho}_{J}=\frac{1}{Z_{J}} \exp [(-\widehat{H}+\mu \widehat{Q}) / T] \mathrm{P}_{\mathbf{J}} \mathrm{P}_{V}
$$

$\mathrm{P}_{\mathbf{J}}$ being the quantum projector onto states with definite angular momentum. As argued in Sect. II, the two ensembles are equivalent for large $J$ and $V$ provided that $\omega / T$ is sufficiently smaller than 1 . Henceforth, we will confine our attention to this case and we will work in the rotational grand-canonical ensemble described by (44).

In the Boltzmann limit of the ideal relativistic quantum gas, all particles can be handled as independent distinguishable objects. Thus, the density operator (44) factorize and we can calculate the polarization by considering the single-particle density operator:

$$
\widehat{\rho}_{\omega}=\frac{1}{z_{\omega}} \exp [(-\widehat{h}+\mu \widehat{q}+\boldsymbol{\omega} \cdot \widehat{\mathbf{j}}) / T] \mathrm{P}_{V}
$$

where $z_{\omega}$ is the single particle partition function:

$$
z_{\omega}=\frac{\lambda}{(2 \pi)^{3}} \int_{V} \mathrm{~d}^{3} \mathrm{x} \int \mathrm{d}^{3} \mathrm{pe}^{-\varepsilon / T} \mathrm{e}^{\boldsymbol{\omega} \cdot(\mathbf{x} \times \mathbf{p}) / T} \operatorname{tr} D^{S}\left(\mathrm{R}_{\hat{\boldsymbol{\omega}}}(i \omega / T)\right)
$$

ensuring the correct normalization $\operatorname{tr} \rho_{\omega}=1$ (this in fact requires $\omega / T \ll 1$, see discussion at the end of Sect. II).

The density operator restricted to the spin degrees of freedom is a function of momentum and reads:

$$
\widehat{\rho}_{\omega}(p)=\frac{1}{\frac{\lambda}{(2 \pi)^{3}} \int_{V} \mathrm{~d}^{3} \mathrm{x}^{-\varepsilon / T} \mathrm{e}^{\boldsymbol{\omega} \cdot(\mathbf{x} \times \mathbf{p}) / T} \operatorname{tr} D^{S}\left(\mathrm{R}_{\hat{\boldsymbol{\omega}}}(i \omega / T)\right)} \exp [(-\widehat{h}+\mu \widehat{q}+\boldsymbol{\omega} \cdot \widehat{\mathbf{j}}) / T] \mathrm{P}_{V}
$$

The polarization is, by definition, the trace of the density matrix $\rho(p)$ multiplied by a suitable spin operator. In non-relativistic quantum mechanics, this is obviously the spin vector operator $\widehat{\mathbf{S}}$ and the polarization can be obtained straightforwardly:

$$
\boldsymbol{\Pi}=\operatorname{tr}\left[\widehat{\mathbf{S}} \widehat{\rho}_{\omega}(p)\right]=\frac{\sum_{n=-S}^{S} n \mathrm{e}^{n \omega / T}}{\sum_{n=-S}^{S} \mathrm{e}^{n \omega / T}} \hat{\boldsymbol{\omega}}
$$

which turns out to be momentum-independent. In relativistic quantum mechanics the proper generalization of the spin angular momentum is the Pauli-Lubanski vector multiplied by $1 / \mathrm{m}$ :

$$
\widehat{W}_{\mu}=-(1 / 2) \sum_{\nu \rho \sigma} \epsilon_{\mu \nu \rho \sigma} \widehat{J}^{\nu \rho} \widehat{P}^{\sigma}
$$

which in fact reduces to the spin operator in the particle rest frame; $\widehat{J}^{\nu \rho}$ are the generators of the Lorentz group. The polarization is thus a four-vector П:

$$
\Pi(p)=\frac{1}{m} \operatorname{tr}_{p}(\widehat{W} \widehat{\rho}(p))
$$

where the trace is to be calculated by summing only over spin degrees of freedom keeping the four-momentum $p$ fixed. This four-vector $\Pi(p)$ has vanishing time component in the particle rest frame, as it is apparent from (50). Before working out eq. (51), we should introduce some important notions about the construction of physical states. We will stick to the notation of ref. [11].

As it is well known, the Pauli-Lubanski vector fulfills the commutation relations:

$$
\begin{aligned}
& {\left[\widehat{W}_{\mu}, \widehat{P}_{\nu}\right]=0} \\
& {\left[\widehat{W}_{\mu}, \widehat{W}_{\nu}\right]=-i \sum_{\rho \sigma} \epsilon_{\mu \nu \rho \sigma} \widehat{W}^{\rho} \widehat{P}^{\sigma}} \\
& \widehat{W} \cdot \widehat{P}=0
\end{aligned}
$$

Hence, if the ket $|p\rangle$ is an eigenvector of $\widehat{P}$, so is $\widehat{W}|p\rangle$. The restriction of $\widehat{W}$ to the eigenspace labelled by fourmomentum $P$ is defined as $\widehat{W}(p)$. Since $\widehat{W}(p) \cdot p=0$, this four-vector operator can be decomposed onto three 
orthonormal spacelike four-vectors $n_{1}(p), n_{2}(p), n_{3}(p)$ which form a basis of the Minkowski space with the unit vector $\hat{p}=p / \sqrt{p^{2}}$ :

$$
\widehat{W}(p)=\sum_{i=1}^{3} \widehat{W}_{i}(p) n_{i}(p)
$$

It can be shown that the operators:

$$
\widehat{S}_{i}(p)=\widehat{W}_{i}(p) / m
$$

form an $\mathrm{SU}(2)$ algebra and are the actual relativistic generalization of the spin angular momentum. The third component $\widehat{S}_{3}(p)$ can be diagonalized along with $\widehat{S}^{2}=-\widehat{W}^{2} / m^{2}$ which is a Casimir of the full group IO $(1,3)^{\uparrow}$, with corresponding eigenvalues $\sigma$ and $S(S+1)$. With a suitable choice of $n_{i}(p)$, i.e.:

$$
n_{i}(p)=[p] e_{i} \quad[p] \equiv \mathrm{R}_{3}(\varphi) \mathrm{R}_{2}(\theta) \mathrm{L}_{3}(\xi)
$$

$e_{i}$ being the unit vectors of spacial axes and $[p]$ being a Lorentz transformation bringing the timelike vector $p_{0}=$ $(m, 0,0,0)$ into the four-momentum $p$ with polar coordinates $\xi, \theta, \varphi$; the eigenvalue $\lambda$ has the physical meaning of the component of intrinsic angular momentum in the rest frame along the direction of particle momentum p. Thus, with the choice (55), $\lambda$ is the helicity in the rest frame and $J$ is, by definition, the spin of the particle. Since, from eqs. (54), (53) and (50) $\widehat{S}_{i}\left(p_{0}\right)=\widehat{J}_{i}$, the spin operators in the rest frame coincide with the generators of the rotation groups. Finally, single particle states will be written as $|p, \sigma\rangle$ with:

$$
\widehat{P}|p, \sigma\rangle=p|p, \sigma\rangle \quad \text { and } \quad \widehat{S}_{3}(p)|p, \sigma\rangle=\sigma|p, \sigma\rangle
$$

and normalization:

$$
\langle p, \sigma \mid q, \tau\rangle=\delta^{3}(\mathbf{p}-\mathbf{q}) \delta_{\sigma \tau}
$$

while the transformation of a state $|p, \sigma\rangle$ under a general Lorentz transformation $\Lambda$ reads:

$$
\widehat{\Lambda}|p, \sigma\rangle=\sum_{\tau}|\Lambda p, \tau\rangle D_{\tau \sigma}^{S}\left([\Lambda p]^{-1} \Lambda[p]\right) \sqrt{\frac{(\Lambda p)^{0}}{p^{0}}} .
$$

We are now in a position to develop eq. (51). By using (53) and (54) we get:

$$
\Pi(p)=\frac{1}{m} \sum_{\sigma, \sigma^{\prime}}\left\langle p, \sigma|\widehat{W}| p, \sigma^{\prime}\right\rangle\left\langle p, \sigma^{\prime}\left|\widehat{\rho}_{\omega}(p)\right| p, \sigma\right\rangle=\sum_{i=1}^{3} \sum_{\sigma, \sigma^{\prime}}\left\langle p, \sigma\left|\widehat{S}_{i}(p)\right| p, \sigma^{\prime}\right\rangle\left\langle p, \sigma^{\prime}\left|\widehat{\rho}_{\omega}(p)\right| p, \sigma\right\rangle n_{i}(p)
$$

Two different matrices appear in the above equation. By definition:

$$
\left\langle p, \sigma\left|\widehat{S}_{i}(p)\right| p, \sigma^{\prime}\right\rangle=D_{\sigma \sigma^{\prime}}^{S}\left(J_{i}\right)
$$

are the matrices of the $\mathrm{SU}(2)$ generators $J_{i}$ in the representation labeled by the particle spin $S$. Furthermore, according to $(48)$ :

$$
\left\langle p, \sigma^{\prime}\left|\widehat{\rho}_{\omega}(p)\right| p, \sigma\right\rangle=\frac{1}{\frac{1}{(2 \pi)^{3}} \int_{V} \mathrm{~d}^{3} \mathrm{x} \mathrm{e}^{\boldsymbol{\omega} \cdot(\mathbf{x} \times \mathbf{p}) / T} \operatorname{tr} D^{S}\left(\mathrm{R}_{\hat{\boldsymbol{\omega}}}(i \omega / T)\right)}\left\langle p, \sigma^{\prime}\left|\exp [\boldsymbol{\omega} \cdot \widehat{\mathbf{j}} / T] \mathrm{P}_{V}\right| p, \sigma\right\rangle
$$

As to the rightmost matrix element, we will restore the vector $\boldsymbol{\phi}=i \boldsymbol{\omega} / T$ and calculate it for real $\boldsymbol{\phi}$ (or imaginary $\boldsymbol{\omega}$ ), then making an analytic continuation to imaginary $\phi$, the same way we did at the end of Sect. II. Therefore:

$$
\left\langle p, \sigma^{\prime}\left|\exp [\boldsymbol{\omega} \cdot \widehat{\mathbf{j}} / T] \mathrm{P}_{V}\right| p, \sigma\right\rangle=\left\langle p, \sigma^{\prime}\left|\exp [-i \boldsymbol{\phi} \cdot \widehat{\mathbf{j}}] \mathrm{P}_{V}\right| p, \sigma\right\rangle=\left\langle p, \sigma^{\prime}\left|\widehat{\mathrm{R}}_{\hat{\boldsymbol{\phi}}}(\phi) \mathrm{P}_{V}\right| p, \sigma\right\rangle
$$

The latter matrix element has been calculated in ref. [3] ${ }^{3}$ :

$$
\begin{aligned}
&\left\langle p, \sigma^{\prime}\left|\widehat{\mathrm{R}}_{\hat{\boldsymbol{\phi}}}(\phi) \mathrm{P}_{V}\right| p, \sigma\right\rangle= \int \mathrm{d}^{3} \mathrm{p}^{\prime} \delta^{3}\left(\mathrm{R}_{\hat{\boldsymbol{\phi}}}(\phi) \mathbf{p}^{\prime}-\mathbf{p}\right) \frac{1}{(2 \pi)^{3}} \int_{V} \mathrm{~d}^{3} \mathrm{x} \mathrm{e}^{i \mathbf{x} \cdot\left(\mathbf{p}-\mathbf{p}^{\prime}\right)} \\
& \times \frac{1}{2}\left(D^{S}\left([p]^{-1} \mathrm{R}_{\hat{\boldsymbol{\phi}}}(\phi)[p]\right)+D^{S}\left([p]^{\dagger} \mathrm{R}_{\hat{\boldsymbol{\phi}}}(\phi)[p]^{\dagger-1}\right)\right)_{\sigma^{\prime} \sigma}\left\langle 0\left|\mathrm{P}_{V}\right| 0\right\rangle \\
&=\frac{1}{(2 \pi)^{3}} \int_{V} \mathrm{~d}^{3} \mathrm{x}^{i \mathbf{x} \cdot\left(\mathbf{p}-\mathrm{R}_{\hat{\boldsymbol{\phi}}}(\phi)^{-1} \mathbf{p}\right)} \frac{1}{2}\left(D^{S}\left([p]^{-1} \mathrm{R}_{\hat{\boldsymbol{\phi}}}(\phi)[p]\right)+D^{S}\left([p]^{\dagger} \mathrm{R}_{\hat{\boldsymbol{\phi}}}(\phi)[p]^{\dagger-1}\right)\right)_{\sigma^{\prime} \sigma}\left\langle 0\left|\mathrm{P}_{V}\right| 0\right\rangle
\end{aligned}
$$

\footnotetext{
${ }^{3}$ It can be easily obtained from eq. (55) in the reference
} 
In this equation, $[p]$ is now meant as an element of the universal covering group of Lorentz group, i.e. SL(2,C), so that the notation $[p]^{\dagger}$ becomes meaningful as well as its finite-dimensional representation matrix $D^{S}([p])$ corresponding to the Lorentz group representation usually labelled as $(S, 0)$ (the $(0, S)$ being the $\left.D^{S}([p])^{\dagger-1}\right)$. The factor $\left\langle 0\left|\mathrm{P}_{V}\right| 0\right\rangle$ is immaterial and also becomes 1 in the large volume limit as $\mathrm{P}_{V} \rightarrow \mathrm{I}$.

If $\phi \ll 1$, as it was supposed to be at the beginning of this section, then:

$$
i \mathbf{x} \cdot\left(\mathbf{p}-\mathrm{R}_{\hat{\phi}}(\phi)^{-1} \mathbf{p}\right) \simeq-i \phi \cdot(\mathbf{x} \times \mathbf{p})
$$

and we can rewrite eq. (63) as:

$$
\left\langle p, \sigma^{\prime}\left|\widehat{\mathrm{R}}_{\hat{\boldsymbol{\phi}}}(\phi) \mathrm{P}_{V}\right| p, \sigma\right\rangle \simeq \frac{1}{(2 \pi)^{3}} \int_{V} \mathrm{~d}^{3} \mathrm{x}^{-i \boldsymbol{\phi} \cdot(\mathbf{x} \times \mathbf{p})} \frac{1}{2}\left(D^{S}\left([p]^{-1} \mathrm{R}_{\hat{\boldsymbol{\phi}}}(\phi)[p]\right)+D^{S}\left([p]^{\dagger} \mathrm{R}_{\hat{\boldsymbol{\phi}}}(\phi)[p]^{\dagger-1}\right)\right)_{\sigma^{\prime} \sigma}
$$

We can now make an analytical continuation to imaginary $\boldsymbol{\phi}$ or real $\boldsymbol{\omega}$ and obtain, by using (61) and (64):

$$
\left\langle p, \sigma^{\prime}\left|\widehat{\rho}_{\omega}(p)\right| p, \sigma\right\rangle=\frac{1}{2 \operatorname{tr} D^{S}\left(\mathrm{R}_{\hat{\boldsymbol{\omega}}}(i \omega / T)\right)}\left(D^{S}\left([p]^{-1} \mathrm{R}_{\hat{\boldsymbol{\omega}}}(i \omega / T)[p]\right)+D^{S}\left([p]^{\dagger} \mathrm{R}_{\hat{\boldsymbol{\omega}}}(i \omega / T)[p]^{\dagger-1}\right)\right)_{\sigma^{\prime} \sigma}
$$

This matrix is hermitian and has trace equal to 1 , as required for a good density matrix. Hermiticity can be shown by taking advantage of a remarkable feature of $\mathrm{SL}(2, \mathrm{C})$ representation [11]:

$$
D^{S}\left(A^{\dagger}\right)=D^{S}(A)^{\dagger}
$$

and taking into account that $D^{S}\left(\mathrm{R}_{\hat{\omega}}(i \omega / T)\right)$ is hermitian.

Plugging (65) into (59), using (60) and carrying out the momentum integration, the final expression of the polarization four-vector for a particle of momentum $p$ in the observer frame is finally achieved:

$$
\begin{aligned}
\Pi(p)^{\mu} & =\frac{1}{2 \operatorname{tr} D^{S}\left(\mathrm{R}_{\hat{\boldsymbol{\omega}}}(i \omega / T)\right)} \sum_{i=1}^{3} \operatorname{tr}\left[D^{S}\left(J_{i}[p]^{-1} \mathrm{R}_{\hat{\boldsymbol{\omega}}}(i \omega / T)[p]\right)+D^{S}\left(J_{i}[p]^{\dagger} \mathrm{R}_{\hat{\boldsymbol{\omega}}}(i \omega / T)[p]^{\dagger-1}\right)\right] n_{i}(p) \\
& =\frac{1}{2 \operatorname{tr} D^{S}\left(\mathrm{R}_{\hat{\boldsymbol{\omega}}}(i \omega / T)\right)} \sum_{i=1}^{3} \operatorname{tr}\left[D^{S}\left(J_{i}[p]^{-1} \mathrm{R}_{\hat{\boldsymbol{\omega}}}(i \omega / T)[p]\right)+D^{S}\left(J_{i}[p]^{\dagger} \mathrm{R}_{\hat{\boldsymbol{\omega}}}(i \omega / T)[p]^{\dagger-1}\right)\right]\left([p] e_{i}\right)^{\mu}
\end{aligned}
$$

The proper polarization four-vector, in the particle rest-frame, has components $\left([p]^{-1} \Pi(p)\right)^{\mu}$, that is:

$$
\Pi_{0}(p)^{\mu}=\frac{1}{2 \operatorname{tr} D^{S}\left(\mathrm{R}_{\hat{\boldsymbol{\omega}}}(i \omega / T)\right)} \sum_{i=1}^{3} \operatorname{tr}\left[D^{S}\left(J_{i}[p]^{-1} \mathrm{R}_{\hat{\boldsymbol{\omega}}}(i \omega / T)[p]\right)+D^{S}\left(J_{i}[p]^{\dagger} \mathrm{R}_{\hat{\boldsymbol{\omega}}}(i \omega / T)[p]^{\dagger-1}\right)\right] \delta_{i}^{\mu}
$$

which has vanishing time component, as required.

The equations (66) and (67) are the general analytical expression of the polarization for a particle with spin $S$. We will now develop them for the most interesting cases of spin $1 / 2$ and spin 1 . Before doing this, we first observe that the Lorentz transformation $[p]$ to calculate the polarization can be chosen arbitrarily. In fact, if $[p]^{\prime} \neq[p]$ also transforms the timelike vector $p_{0}=(m, 0,0,0)$ into $p$, then $[p]^{-1}[p]^{\prime}$ is a pure rotation $\mathrm{R}$ as it leaves $p_{0}$ invariant. Hence, the polarization four-vector $\Pi(p)$ defined with $[p]^{\prime}$ becomes:

$$
\begin{aligned}
\Pi(p) & \propto \sum_{i=1}^{3} \frac{1}{2} \operatorname{tr}\left[D^{S}\left(J_{i}[p]^{\prime-1} \mathrm{R}_{\hat{\boldsymbol{\omega}}}(i \omega / T)[p]^{\prime}\right)+D^{S}\left(J_{i}[p]^{\prime \dagger} \mathrm{R}_{\hat{\boldsymbol{\omega}}}(i \omega / T)[p]^{\prime \dagger-1}\right)\right]\left([p]^{\prime} e_{i}\right) \\
& =\sum_{i=1}^{3} \frac{1}{2} \operatorname{tr}\left[D^{S}\left(J_{i} \mathrm{R}^{-1}[p]^{-1} \mathrm{R}_{\hat{\boldsymbol{\omega}}}(i \omega / T)[p] \mathrm{R}\right)+D^{S}\left(J_{i} \mathrm{R}^{-1}[p]^{\dagger} \mathrm{R}_{\hat{\boldsymbol{\omega}}}(i \omega / T)[p]^{\dagger-1} \mathrm{R}\right)\right]\left([p] \mathrm{R}_{i}\right) \\
& =\sum_{i=1}^{3} \frac{1}{2} \operatorname{tr}\left[D^{S}\left(\mathrm{R}_{i} \mathrm{R}^{-1}[p]^{-1} \mathrm{R}_{\hat{\boldsymbol{\omega}}}(i \omega / T)[p]\right)+D^{S}\left(\mathrm{R}_{i} \mathrm{R}^{-1}[p]^{\dagger} \mathrm{R}_{\hat{\boldsymbol{\omega}}}(i \omega / T)[p]^{\dagger-1}\right)\right]\left([p] \mathrm{R}_{i}\right) \\
& =\sum_{i=1}^{3} \frac{1}{2} \operatorname{tr}\left[D^{S}\left(J_{\operatorname{Re}_{i}}[p]^{-1} \mathrm{R}_{\hat{\boldsymbol{\omega}}}(i \omega / T)[p]\right)+D^{S}\left(J_{\operatorname{Re}_{i}}[p]^{\dagger} \mathrm{R}_{\hat{\boldsymbol{\omega}}}(i \omega / T)[p]^{\dagger-1}\right)\right]\left([p] \mathrm{R}_{i}\right)
\end{aligned}
$$

where we used the unitarity of R. The last expression in (68) is apparently equal to the one in (66). 


\section{A. $\quad$ Spin $S=1 / 2$}

Choosing for $[p]$ a pure Lorentz boost, we have [11]:

$$
D^{1 / 2}([p])=D^{1 / 2}([p])^{\dagger}=\frac{m+\varepsilon+\boldsymbol{\sigma} \cdot \mathbf{p}}{\sqrt{2 m(m+\varepsilon)}}
$$

and

$$
D^{1 / 2}([p])^{-1}=D^{1 / 2}([p])^{\dagger-1}=\frac{m+\varepsilon-\boldsymbol{\sigma} \cdot \mathbf{p}}{\sqrt{2 m(m+\varepsilon)}}
$$

where $\boldsymbol{\sigma}$ are the Pauli matrices. Therefore:

$$
\left.\operatorname{tr}\left[D^{1 / 2}\left(J_{i}[p]^{-1} \mathrm{R}_{\hat{\boldsymbol{\omega}}}(i \omega / T)[p]\right)\right]=\operatorname{tr}\left[\frac{\sigma_{i}}{2} \frac{m+\varepsilon-\boldsymbol{\sigma} \cdot \mathbf{p}}{\sqrt{2 m(m+\varepsilon)}} \mathbf{R}_{\hat{\boldsymbol{\omega}}}(i \omega / T) \frac{m+\varepsilon+\boldsymbol{\sigma} \cdot \mathbf{p}}{\sqrt{2 m(m+\varepsilon)}}\right)\right]
$$

whereas, because of (69) and (70):

$$
\left.\operatorname{tr}\left[D^{1 / 2}\left(J_{i}[p]^{\dagger} \mathrm{R}_{\hat{\boldsymbol{\omega}}}(i \omega / T)[p]^{\dagger-1}\right)\right]=\operatorname{tr}\left[\frac{\sigma_{i}}{2} \frac{m+\varepsilon+\boldsymbol{\sigma} \cdot \mathbf{p}}{\sqrt{2 m(m+\varepsilon)}} \mathrm{R}_{\hat{\boldsymbol{\omega}}}(i \omega / T) \frac{m+\varepsilon-\boldsymbol{\sigma} \cdot \mathbf{p}}{\sqrt{2 m(m+\varepsilon)}}\right)\right]
$$

i.e. it is obtained from (71) by reflecting p. Since:

$$
D^{1 / 2}\left(\mathrm{R}_{\hat{\boldsymbol{\omega}}}(i \omega / T)\right)=\mathrm{I} \cos \frac{i \omega}{2 T}-i \boldsymbol{\sigma} \cdot \hat{\boldsymbol{\omega}} \sin \frac{i \omega}{2 T}=\mathrm{I} \cosh \frac{\omega}{2 T}+\boldsymbol{\sigma} \cdot \hat{\boldsymbol{\omega}} \sinh \frac{\omega}{2 T}
$$

we have, for $(71)$

$$
\begin{aligned}
& \frac{m+\varepsilon-\boldsymbol{\sigma} \cdot \mathbf{p}}{\sqrt{2 m(m+\varepsilon)}}\left(\mathrm{I} \cosh \frac{\omega}{2 T}+\boldsymbol{\sigma} \cdot \hat{\boldsymbol{\omega}} \sinh \frac{\omega}{2 T}\right) \frac{m+\varepsilon+\boldsymbol{\sigma} \cdot \mathbf{p}}{\sqrt{2 m(m+\varepsilon)}} \\
& =\mathrm{I} \cosh \frac{\omega}{2 T}+\sinh \frac{\omega}{2 T}\left[\frac{m+\varepsilon}{2 m} \boldsymbol{\sigma} \cdot \hat{\boldsymbol{\omega}}+\frac{i}{m} \boldsymbol{\sigma} \cdot(\hat{\boldsymbol{\omega}} \times \mathbf{p})-\frac{\hat{\boldsymbol{\omega}} \cdot \mathbf{p} \boldsymbol{\sigma} \cdot \mathbf{p}-\boldsymbol{\sigma} \cdot(\mathbf{p} \times(\hat{\boldsymbol{\omega}} \times \mathbf{p}))}{2 m(\varepsilon+m)}\right]
\end{aligned}
$$

Now we have to take the sum of the two traces (71) and (72) implying that all terms which change sign in a reflection of the momentum $\mathbf{p}$ in (74) vanish. Therefore, we are left with:

$$
\begin{aligned}
& 2 \operatorname{tr}\left\{\frac{\sigma_{i}}{2}\left[\mathrm{I} \cosh \frac{\omega}{2 T}+\sinh \frac{\omega}{2 T}\left[\frac{m+\varepsilon}{2 m} \boldsymbol{\sigma} \cdot \hat{\boldsymbol{\omega}}-\frac{\hat{\boldsymbol{\omega}} \cdot \mathbf{p} \boldsymbol{\sigma} \cdot \mathbf{p}-\boldsymbol{\sigma} \cdot(\mathbf{p} \times(\hat{\boldsymbol{\omega}} \times \mathbf{p}))}{2 m(\varepsilon+m)}\right]\right]\right\} \\
& =\sinh \frac{\omega}{2 T}\left[\frac{m+\varepsilon}{m} \hat{\boldsymbol{\omega}}_{i}-\frac{\hat{\boldsymbol{\omega}} \cdot \mathbf{p p}_{i}-(\mathbf{p} \times(\hat{\boldsymbol{\omega}} \times \mathbf{p}))_{i}}{m(\varepsilon+m)}\right]
\end{aligned}
$$

Taking into account that:

$$
\operatorname{tr} D^{1 / 2}\left(\mathrm{R}_{\hat{\boldsymbol{\omega}}}(i \omega / T)\right)=2 \cosh \frac{\omega}{2 T}
$$

we can obtain the polarization vector in the rest-frame putting (75) and (76) into eq. (67):

$$
\begin{aligned}
\mathbf{\Pi}_{0} & =\frac{1}{4} \tanh \frac{\omega}{2 T}\left[\frac{m+\varepsilon}{m} \hat{\boldsymbol{\omega}}-\frac{\hat{\boldsymbol{\omega}} \cdot \mathbf{p p}-\mathbf{p} \times(\hat{\boldsymbol{\omega}} \times \mathbf{p})}{m(\varepsilon+m)}\right] \\
& =\frac{1}{4} \tanh \frac{\omega}{2 T}\left[\frac{m+\varepsilon}{m} \hat{\boldsymbol{\omega}}-\frac{\hat{\boldsymbol{\omega}} \cdot \mathbf{p} \mathbf{p}-\mathrm{p}^{2} \hat{\boldsymbol{\omega}}+\hat{\boldsymbol{\omega}} \cdot \mathbf{p} \mathbf{p}}{m(\varepsilon+m)}\right] \\
& =\frac{1}{2} \tanh \frac{\omega}{2 T}\left[\frac{\varepsilon}{m} \hat{\boldsymbol{\omega}}-\frac{\hat{\boldsymbol{\omega}} \cdot \mathbf{p p}}{m(\varepsilon+m)}\right]
\end{aligned}
$$

Therefore, unlike in the non-relativistic case, the polarization vector has a component along particle momentum in the observer frame. This effect is owing to the vector nature of the polarization; indeed, the components of polarization in the observer frame (66) can be obtained with a general Lorentz boost [12]:

$$
\boldsymbol{\Pi}=\boldsymbol{\Pi}_{0}+\frac{\gamma^{2}}{\gamma+1} \boldsymbol{\beta} \cdot \boldsymbol{\Pi}_{0} \boldsymbol{\beta} \quad \Pi^{0}=\gamma \boldsymbol{\beta} \cdot \boldsymbol{\Pi}_{0}
$$


with $\boldsymbol{\beta}=\mathbf{p} / \varepsilon$ and $\gamma=\varepsilon / m$. Therefore, by using (77), eq. (78) becomes:

$$
\boldsymbol{\Pi}=\frac{1}{2} \tanh \frac{\omega}{2 T} \frac{\varepsilon}{m} \hat{\boldsymbol{\omega}} \quad \Pi^{0}=\frac{1}{2} \tanh \frac{\omega}{2 T} \frac{\hat{\boldsymbol{\omega}} \cdot \mathbf{p}}{m}
$$

Hence, the polarization three-vector is aligned with the angular velocity in the observer frame and not in the particle frame. Yet, there is also a time component which vanishes in the non-relativistic limit $\mathbf{p} / m \rightarrow 0$, where the (79) correctly yields (49). The remarkable difference with respect to the non-relativistic case (49) is that polarization now depends on momentum. The longitudinal component, along $\mathbf{p}$, is:

$$
\Pi_{0} \cdot \hat{\mathbf{p}}=\frac{1}{2} \tanh \frac{\omega}{2 T} \hat{\mathbf{p}} \cdot \hat{\boldsymbol{\omega}}
$$

while the component along the rotation axis turns out to be:

$$
\boldsymbol{\Pi}_{0} \cdot \hat{\boldsymbol{\omega}}=\frac{1}{2} \tanh \frac{\omega}{2 T}\left[\frac{\varepsilon}{m}-\frac{\mathrm{p}^{2}(\hat{\boldsymbol{\omega}} \cdot \hat{\mathbf{p}})^{2}}{m(\varepsilon+m)}\right]
$$

which shows a very interesting feature: the polarization is maximal for particles with momentum orthogonal to the rotation axis. Furthermore, the polarization increases with energy, being proportional to $\varepsilon / m$. Of course this behaviour cannot go on indefinitely because polarization cannot exceed $1 / 2$ in any direction; since $\omega / T \ll 1$, it is seen from eq. (81) that something must happen when $\varepsilon \sim 2 m T / \omega$. Indeed, we have pointed out in Sect. IV that when momentum is of the order of $J / R$, the saddle point expansion of partition function at fixed $J$ can no longer be independent of $\mathrm{p}$, and $\omega$ becomes in fact a function of $p$, that is $\omega(p)$, for the spectrum (32) expansion. This has some impact on the polarization vector: at some large momentum the dependence of $\omega$ on $p$ should restore the natural $1 / 2$ bound.

\section{B. Spin $\mathrm{S}=1$}

The calculation of the polarization for massive spin 1 particles is more involved than for spin $1 / 2$ and we have carried it out by performing explicitely the multiplication of matrices of $\mathrm{SL}(2, \mathrm{C}) D^{1}$ representation. Since eventually one has to calculate traces, the choice of the basis for the matrices is arbitrary and we have written $[p]=\mathrm{R}_{3}(\varphi) \mathrm{R}_{2}(\theta) \mathrm{L}_{3}(\xi)$, being $\cosh \xi=\varepsilon / m$, in the cartesian basis for the $D^{1}$ representation space:

$$
\begin{aligned}
& D^{1}\left(\mathrm{R}_{3}(\varphi)\right)=\left(\begin{array}{ccc}
\cos \varphi & -\sin \varphi & 0 \\
\sin \varphi & \cos \varphi & 0 \\
0 & 0 & 1
\end{array}\right) \quad D^{1}\left(\mathrm{R}_{2}(\theta)\right)=\left(\begin{array}{ccc}
\cos \theta & 0 & \sin \theta \\
0 & 1 & 0 \\
-\sin \theta & 0 & \cos \theta
\end{array}\right) \\
& D^{1}\left(\mathrm{~L}_{3}(\xi)\right)=\exp \left[-i \xi K_{3}\right]=\exp \left[\xi J_{3}\right]=D^{1}\left(\mathrm{R}_{3}(i \xi)\right)=\left(\begin{array}{ccc}
\cosh \xi & -i \sinh \xi & 0 \\
i \sinh \xi & \cosh \xi & 0 \\
0 & 0 & 1
\end{array}\right) \\
& D^{1}\left(\mathrm{R}_{\hat{\boldsymbol{\omega}}}(i \omega / T)\right)=\exp \left[\hat{\boldsymbol{\omega}} / T \cdot D^{1}(\mathbf{J})\right] \quad D^{1}\left(J_{i}\right)_{j k}=-i \epsilon_{i j k}
\end{aligned}
$$

Multiplying the matrices in eq. (82) and calculating the traces, according to eq. (67), one gets:

$$
\boldsymbol{\Pi}_{0}=\frac{2 \sinh (\omega / T)}{2 \cosh (\omega / T)+1}\left[\frac{\varepsilon}{m} \hat{\boldsymbol{\omega}}-\frac{\hat{\boldsymbol{\omega}} \cdot \mathbf{p p}}{m(\varepsilon+m)}\right]
$$

where the denominator $2 \cosh (\omega / T)+1$ is the trace of the matrix $D^{1}\left(\mathrm{R}_{\hat{\boldsymbol{\omega}}}(i \omega / T)\right)$. Therefore, the kinematical structure of the polarization vector is the same as in the spin $1 / 2$ case, which is a reasonable outcome; in fact, the properties of Lorentz transformation of a polarization vector should not depend on the particle spin itself.

In the case of spin 1 it is also interesting to calculate the fraction of the 00 component of the density matrix (65). The calculation can be done quickly by noting that:

$$
\rho_{\omega 00}(p)=\operatorname{tr} P_{3} \widehat{\rho}=\sum_{\sigma}\left\langle p, \sigma\left|P_{3} \widehat{\rho}_{\omega}(p)\right| p, \sigma\right\rangle
$$

$P_{3}$ being the projector onto the state $|p, 0\rangle$. Written in the cartesian basis the matrix corresponding to $P_{3}$ is simply:

$$
P_{3}=\left(\begin{array}{lll}
0 & 0 & 0 \\
0 & 0 & 0 \\
0 & 0 & 1
\end{array}\right)
$$


because of the choice of $z$ axis as quantization axis, according to (56) Therefore, by plugging eq. (65) into eq. (84):

$$
\rho_{\omega 00}(p)=\frac{1}{2 \operatorname{tr} D^{1}\left(\mathrm{R}_{\hat{\boldsymbol{\omega}}}(i \omega / T)\right)} \operatorname{tr}\left[P_{3} D^{1}\left([p]^{-1} \mathrm{R}_{\hat{\boldsymbol{\omega}}}(i \omega / T)[p]\right)+P_{3} D^{1}\left([p]^{\dagger} \mathrm{R}_{\hat{\boldsymbol{\omega}}}(i \omega / T)[p]^{\dagger-1}\right)\right]
$$

yielding:

$$
\rho_{\omega 00}(p)=\frac{1}{2 \cosh (\omega / T)+1}\left[\cosh (\omega / T)+\frac{(\mathbf{p} \cdot \boldsymbol{\omega})^{2}}{\mathrm{p}^{2} \omega^{2}}(1-\cosh (\omega / T))\right]
$$

For small $\omega / T$, we have:

$$
\rho_{\omega 00}(p) \simeq \frac{1}{3}+\frac{1-3(\hat{\mathbf{p}} \cdot \hat{\boldsymbol{\omega}})^{2}}{18} \frac{\omega^{2}}{T^{2}}
$$

\section{Discussion}

In both spin $1 / 2$ and spin 1 cases, we have seen that the proper polarization vector has the same structure, so that we can easily generalize the above results to any spin:

$$
\boldsymbol{\Pi}_{0}=\frac{\sum_{n=-S}^{S} n \mathrm{e}^{n \omega / T}}{\sum_{n=-S}^{S} \mathrm{e}^{n \omega / T}}\left[\frac{\varepsilon}{m} \hat{\boldsymbol{\omega}}-\frac{\hat{\boldsymbol{\omega}} \cdot \mathbf{p p}}{m(\varepsilon+m)}\right]
$$

This non-vanishing polarization means that spin states are not evenly populated in an equilibrated thermodynamical system with finite, macroscopic, angular momentum. Of course reaching equilibrium for the spin degrees of freedom implies that a small interaction should exists involving particle spin, e.g. a spin-orbit coupling. Yet, for the ideal relativistic gas, this interaction is assumed to be negligible in comparison with the pure kinematical effect of angular momentum conservation. This can be rephrased in the rotating frame as follows: the actual interaction involving spin is negligible with respect to the spin-rotation coupling. Since a macroscopic thermodynamical system can be conceptually divided into elementary fluid cells, and each cell participating in the rigid rotation is an accelerated system, for our result to be consistent with locality, one has to conclude that, in general, acceleration involves a polarization expressed by (89) where $\boldsymbol{\omega}$ is to be interpreted as a local vector field involving local quantities such as velocity and acceleration of the cell.

\section{Acknowledgments}

We are grateful for interesting discussions to R. Jaffe, G. Longhi, L. Lusanna, K. Rajagopal, H. Satz, D. Seminara. We thank Galileo Galilei Institute for hospitality.

\section{APPENDIX A - ENTROPY FOR A SPINNING SYSTEM}

The entropy of a system with finite angular momentum $\mathbf{J}$ can be calculated easily from the expression of the probability of a state with energy $E$, vanishing momentum, charge $Q$ and fixed $\mathbf{J}$ in the grand-canical ensemble:

$$
p=\frac{1}{Z_{J}} \exp [-E / T+\mu Q / T]
$$

so that, by using (14)

$$
S=-\sum_{\text {stateswithfixed } \mathbf{J}} p \log p=\frac{\langle E\rangle}{T}-\frac{\mu\langle Q\rangle}{T}+\log Z_{J}=\frac{U}{T}-\frac{\mu\langle Q\rangle}{T}-\frac{\boldsymbol{\omega} \cdot \mathbf{J}}{T}+\log Z_{\omega}
$$

which is the known expression of entropy for a rotating system. The logarithm of the partition function can then be identified with the integral of the pressure:

$$
\log Z_{\omega}=\frac{1}{T} \int_{V} \mathrm{~d}^{3} \mathrm{x} p(\mathbf{x})
$$


where, unlike in familiar cases, the pressure is not uniform due to rotation. From the entropy expression:

$$
T S=U-\mu\langle Q\rangle-\boldsymbol{\omega} \cdot \mathbf{J}+\int_{V} \mathrm{~d}^{3} \mathrm{x} p(\mathbf{x})
$$

we can derive the relation [5]

$$
\left.\frac{\partial S}{\partial \mathbf{J}}\right|_{V, U, Q}=-\frac{\omega}{T}
$$

\section{APPENDIX B - EQUILIBRIUM CONFIGURATION OF A RELATIVISTIC ROTATING SYSTEM}

We will now show that a relativistic macroscopic system with finite angular momentum at equilibrium must be rigidly rotating by generalizing an argument by Landau [5] for non-relativistic system.

Let us consider a generic isolated hdyrodynamical system with velocities $\mathbf{v}_{i} i$ being the label of hydrodynamical cells. In order to find the equilibrium configuration the entropy should be maximized with the constraint of energy, momentum and angular momentum conservation. Therefore, we have to find the extremum points of:

$$
\sum_{i} S_{i}-\beta\left(\sum_{i} E_{i}-E_{0}\right)+\boldsymbol{\beta} \cdot \sum_{i} \mathbf{P}_{i}+\beta \boldsymbol{\omega} \cdot\left(\sum_{i} \mathbf{x}_{\mathbf{i}} \times \mathbf{P}_{i}-\mathbf{J}\right)
$$

where we have taken the total momentum vanishing, i.e. we are working in the system's rest frame; $E_{0}$ is the total energy and $\mathbf{J}$ the total angular momentum; $\beta, \boldsymbol{\beta}$ and $\boldsymbol{\omega} / T$ are Lagrange multipliers enforcing the conservation laws. Since the entropy is a relativistic invariant, it can only depend on the mass of the cell, i.e. $S_{i}=S_{i}\left(\sqrt{E_{i}^{2}-\mathbf{P}_{i}^{2}}\right)$. In order to find the equilibrium configuration, one has to maximize (95) with respect to each $\mathbf{P}_{i}$ and $E_{i}$. Thus:

$$
\frac{\partial S_{i}}{\partial E_{i}}=\frac{E_{i}}{M_{i}} \frac{\partial S_{i}}{\partial M_{i}}=\beta \quad \forall i
$$

and:

$$
-\frac{\partial S_{i}}{\partial \mathbf{P}_{\mathbf{i}}}=\frac{\mathbf{P}_{i}}{M_{i}} \frac{\partial S_{i}}{\partial M_{i}}=\boldsymbol{\beta}+\beta \boldsymbol{\omega} \times \mathbf{x}_{i} \quad \forall i
$$

Taking into account that $\partial S_{i} / \partial M_{i}$ is the inverse of the proper temperature $T_{i}$ of the $i$ th cell by definition, eq. (96) implies that:

$$
\frac{\gamma_{i}}{T_{i}}=\text { constant }=\beta \equiv \frac{1}{T}
$$

where $T$ is defined the global temperature of the system. Plugging (98) into (97) we get:

$$
\frac{\gamma_{i} \mathbf{v}_{i}}{T_{i}}=\frac{\mathbf{v}_{i}}{T}=\boldsymbol{\beta}+\frac{\boldsymbol{\omega} \times \mathbf{x}_{i}}{T}
$$

For the total momentum to vanish, the vector $\boldsymbol{\beta}$ should be 0 as well and we are left with:

$$
\mathbf{v}_{i}=\boldsymbol{\omega} \times \mathbf{x}_{i}
$$

that is a rigid rotation around the axis $\boldsymbol{\omega}$. To show that $T=1 / \beta$ is in fact the global temperature of the sytem, one has to consider that at equilibrium, using formula (91) in Appendix A for the entropy of the cell $i$ :

$$
S \equiv \sum_{i} S_{i}=\sum_{i} \frac{M_{i}}{T_{i}}+\ldots=\sum_{i} \frac{\gamma_{i} M_{i}}{T}+\ldots=\sum_{i} \frac{E_{i}}{T}=\frac{M}{T}+\ldots
$$

$M$ being the mass of the system, because $\mathbf{P}=0$. Therefore:

$$
\frac{\partial S}{\partial M}=\frac{1}{T}
$$

showing that $T$ is the actual temperature of the system. The relation (98) can be rewritten as, by using (100):

$$
T=T_{i} \sqrt{1-\left\|\boldsymbol{\omega} \times \mathbf{x}_{i}\right\|^{2}}
$$


in accordance with eq. (11). The involved physical meaning is as follows: the temperature measured by a thermometer at rest in the observer frame, is lower than that measured by a comoving thermometer, i.e. at rest in the cell frame. This is a pure relativistic effect which has no correspondance in classical thermodynamics.

The above argument can be extended to include the intrinsic angular momentum contribution $\mathbf{J}_{i}$ of each cell to $\mathbf{J}$. Eq. (95) now reads:

$$
\sum_{i} S_{i}\left(\sqrt{E_{i}^{2}-\mathbf{P}_{i}^{2}}, \mathbf{J}_{i}\right)-\beta\left(\sum_{i} E_{i}-E_{0}\right)+\boldsymbol{\beta} \cdot \sum_{i} \mathbf{P}_{i}+\beta \boldsymbol{\omega} \cdot\left(\sum_{i} \mathbf{x}_{\mathbf{i}} \times \mathbf{P}_{i}+\mathbf{J}_{i}-\mathbf{J}\right)
$$

The conclusions are similar, with the additional condition:

$$
\frac{\partial S_{i}}{\partial \mathbf{J}_{\mathbf{i}}}=-\frac{\boldsymbol{\omega}}{T}
$$

in accordance with eq. (94).

\section{REFERENCES}

[1] L. Moretto, Phys. Rev. C 29, 843 (1984).

[2] A. Botvina, D. H. E. Gross, Nucl. Phys. A 592, 257 (1995).

[3] F. Becattini, L. Ferroni, arXiv:0707.0793, to appear in Eur. Phys. J. C.

[4] P. Castorina, D. Kharzeev and H. Satz, Eur. Phys. J. C 52, 187 (2007)

[5] L. Landau, L. Lifshitz, "Statistical Physics", Pergamon Press, 1980.

[6] B. Touschek, Nuovo Cimento B 63295 (1968).

[7] B. Mashhoon, "Quantum theory in accelerated frames of reference," Lect. Notes Phys. 702, 112 (2006).

[8] W. Israel, Ann. Phys. 100310 (1976).

[9] R. Hagedorn and U. Wambach, Nucl. Phys. B 123, 382 (1977).

[10] B. Mashhoon and H. Kaiser, Physica B 385, 1381 (2006);

J. Anandan, J. Suzuki, "Quantum Mechanics in a Rotating Frame", arXiv:quant-ph/0305081, in Relativity in rotating frames, Kluwer 2003, p. 361;

[11] P. Moussa, R. Stora, Angular Analysis of Elementary Particle Reactions, in Proceedings of the 1966 International School on Elementary Particles, Hercegnovi (Gordon and Breach, New York, London, 1968).

[12] J. D. Jackson, "Classical Electrodynamics", 3rd edition, John Wiley and Sons, 1998. 